\title{
Hepatic Changes Detected by Non-Invasive Biomarker, FIB4 Score and Transient Elastography in Diabetes Mellitus Patients
}

\section{IJCRR}

Section: Healthcare

ISI Impact Factor

(2019-20): 1.628

IC Value (2019): 90.81

SJIF (2020) $=7.893$

(c) (1) (3)

Copyright@IJCRR

\section{Vipin Singh ${ }^{1}$, Balvir Singh ${ }^{2 *}$}

'Junior Resident, P.G. Department of Medicine, S.N. Medical College, Agra, India; ${ }^{2}$ Professor and Head, P.G. Department of Medicine, S.N. Medical College, Agra, India.

\section{ABSTRACT}

Objectives: To study the prevalence of hepatic changes in diabetics, evaluate possible risk factors leading to fibrosis and assess the best non-invasive test in between FIB4 score and TE to diagnose hepatic fibrosis earliest possible.

Methods: The study was conducted on 304 patients, attending outdoor, diabetic clinics, patients admitted with deranged liver function test, ultrasonography finding and clinical findings inwards of post graduate department of medicine, during a period from October 2018 to November 2019. Informed consent was taken from the patients and detailed history physical examination and laboratory investigations were carried out. NAFLD was diagnosed based on TE (FibroScan).

Results: Among patients screened $204(67 \%)$ have developed hepatic involvement in the form of NAFLD picked up by TE, among this $65(32 \%)$ had fatty liver without fibrosis or mild fibrosis. The remaining $139(68 \%)$ were divided into different categories like significant fibrosis in 92 (45\%), severe fibrosis in 16(8\%) and cirrhosis in 31(15\%)patients. The severity of NAFLD was associated with the significant increase in mean age group, mean waist-hip ratio, mean BMI, mean fasting blood sugar, mean $\mathrm{HbA} 1 \mathrm{C}$ and mean triglyceride.

Conclusion: To detect the earliest changes of NAFLD patients of diabetes mellitus should undergo liver function tests, FIB4 scoring, ultrasonography of liver and tissue elastography. Prior detection with the help of these non-invasive tests will help in the prevention of fatal complications like cirrhosis, carcinoma of the liver without the potential risk of liver biopsy in unselected patients and also help in altering the disease course.

Key Words: Diabetes mellitus, FIB4 score, NAFLD, Transientelastography, Ultrasonography, Fasting blood sugar

\section{INTRODUCTION}

In India, 69.1 million people are suffering from diabetes mellitus and it is estimated that India has the second-highest number of cases of diabetes mellitus in the world after China in 2015. ${ }^{1}$ In India, the prevalence of diabetes mellitus ranges from 5 to $17 \%$. Reid et al., have shown that Indians are prone to diabetes mellitus at a much younger age with a greater risk for diabetes mellitus associated complications despite less degree of obesity. ${ }^{2}$ Almost an entire spectrum of liver diseases is noticed in patients with type 2 diabetes mellitus. This comprises abnormal liver enzymes, NAFLD, cirrhosis, hepatocellular carcinoma, and acute liver failure. The prevalence of diabetes in cirrhosis is $12.3-57 \%{ }^{3}$ Hence, patients with diabetes mellitus have a high prevalence of liver disease and patients with the liver disease having a high prevalence of diabetes mellitus.
NAFLD is found to be the most common cause of chronic liver disease in USA. ${ }^{2}$ It is described as presence of fatty liver disease in patients with $<20 \mathrm{gm}$ alcohol/day. NAFLD, which look like alcoholic liver disease, comprises a spectrum of liver diseases from steatosis (fatty infiltration of the liver) to $\mathrm{NASH}$, which involves steatosis plus inflammation, necrosis, and fibrosis. The prevalence of NAFLD in diabetes is estimated at $34-74 \%{ }^{3-7}$ and in diabetes with obesity virtually it's $100 \% .^{13}$ The fundamental issue in the management of patients with NAFLD is the diagnosis of steatohepatitis and fibrosis at an early stage. This would help in the identification of those patients who require strict follow-up, lifestyle changes for weight reduction and management of the metabolic syndrome. ${ }^{8-11}$

Corresponding Author:

Dr. (Prof) Balvir Singh, Professor and Head, P.G. Department of Medicine, Sarojini Naidu medical college, Agra-282002 Uttar Pradesh, India Mobile: +919412722223, Email: tajgastro_bs@rediffmail.com

ISSN: 2231-2196 (Print)

Received: 28.01 .2021
ISSN: 0975-5241 (Online)

Revised: 02.04 .2021
Accepted: 28.05 .2021

Published: 11.10 .2021 
Liver biopsy is the gold standard for diagnosis of NASH and evaluating fibrosis, but due to its limitation of Potential risk of sampling error, inter-intraobserver variability, invasiveness and scares acceptability by the patient and with the high prevalence of hepatic steatosis and its lower risk of progression in the majority of the affected people make liver biopsy an unsuitable tool for diagnosis in an unselected patient. ${ }^{12-15}$

FibroScan with CAP measurement can measure hepatic steatosis levels. Though, TE done by FibroScan is suggested by the AASLD and EASL to measure liver fibrosis in patients with NAFLD. ${ }^{14-15}$ This is a non-invasive simple-to-perform imaging modality with high accuracy to measure liver stiffness and hepatic fat deposition.

Thus, So far there has been little knowledge on the prevalence of NAFLD and liver fibrosis in diabetic populations in India. Therefore, our study is to detect the prevalence of CAP-defined NAFLD and the severity of liver fibrosis detected by TE in patients of diabetes mellitus.

\section{MATERIAL AND METHODS:}

Study population: This was a hospital-based cross-sectional study conducted in the postgraduate department of medicine at Sarojini Naidu medical college, Agra, Uttar Pradesh. The study was done after getting clearance from the Ethical Committee of our institute and permission from the appropriate authority (Institutional ethical committee approval no. IEC/2021/68). The study was conducted for one year with a total of 304 patients attending OPD, diabetic OPD, admitted with deranged liver function test, USG finding and clinical findings in diabetic patients admitted inwards. All 304 patients fulfilled both inclusion criteria (Diabetic type 1 and type 2) and exclusion criteria: ${ }^{16-19}$

i. Excessive daily alcohol intake $\geq 30 \mathrm{~g}$ in men and $\geq 20$ $\mathrm{g}$ in women.

ii. Causes of secondary hepatic steatosis: pregnancy, abuse of steatogenic drugs (amiodarone, tamoxifen, methotrexate, corticosteroid, estrogen) and severe malnutrition.

iii. Positive hepatitis $B$ surface antigen or hepatitis $C$ viral antibody.

iv. Other chronic liver diseases: autoimmune hepatitis, hemochromatosis, Wilson's disease, primary biliary cirrhosis, and drug-induced hepatitis.

Clinical assessment: Patients meeting all study criteria were enrolled and participating subjects who had given their written informed consent were checked for medical histories, physical examinations and laboratory tests. Most patients had the following symptoms: polyuria, polydipsia, polyphagia, right upper quadrant pain/discomfort, right hypochondrial pain on rest or exertion, loss of appetite, postprandial abdominal fullness, increased blood pressure, increased blood triglyceride levels and history of hepatic involvement. In these patients subsequent parameters were documented: Age, sex, BMI, weight, waist circumference, waist-hip ratio, CBC, ALT, AST, GGT, ALP, triglycerides, metabolic syndrome, fasting blood sugar, postprandial blood sugar, $\mathrm{HbAlc}$, as well as the liver aspect on US and LSMby means of TE and FIB4 score. ${ }^{20}$

Ultrasound examination: The severity of fat amount within the liver was evaluated by ultrasound in fasting patients, with a Siemens ultrasound system A semi-quantitative scale was used, according to the subjective assessment of the "echogenicity" of the liver as compared to the renal parenchyma and the intensity of "posterior attenuation". The scale ranged from no steatosis (no posterior attenuation), S1: mild steatosis (discrete posterior attenuation), S2: moderate steatosis (obvious attenuation), S3: severe steatosis (intense posterior attenuation which makes it impossible to visualise the diaphragm). ${ }^{21-26}$

FibroScan Examination: TE was performed by an experienced operator using fibroScan 502 touches with an M probe. LSM and CAP were defined by the median of 10 successful measurements and CAP score was considered consistent if 10 valid readings were obtained. Patients were considered to possess NAFLD based on Fibro Scan if CAP $>233 \mathrm{~dB} / \mathrm{m}^{16}$; absent or mild fibrosis (F0-F1), significant liver fibrosis $(\geq F 2)$, advanced fibrosis $(\geq F 3)$ and cirrhosis (F4) supported TE were defined $<0.75,0.80(0.75-0.84)$ for patients with significant fibrosis $(\mathrm{F}>2), 0.90(0.86-0.93)$ for patients with severe fibrosis (F3), and $0.96(0.94-0.98)$ or higher for patients with cirrhosis, respectively, this range was in line with the previous study of LSM using TE in NAFLD patients ${ }^{17}$. Patients with NAFLD with significant fibrosis were diagnosed with NASH. Fibroscan: Castera transient elastography kPa was $<7.5 \mathrm{~F} 0-\mathrm{F} 1$ absent fibrosis, 7.5-8.5 F2 significant fibrosis, 8.6-9.3 F3 severe fibrosis, $>9.4 \mathrm{~F} 4$ cirrhosis.

FIB4 score: Stages of fibrosis were defined as FIB4 score $>3.25$ or TE value $>9.4 \mathrm{kPa}$ for advanced fibrosis and a FIB4 score $<3.25$ or a TE value $<9.4 \mathrm{kPa}$ for non-advanced fibrosis. Furthermore, the lowest cut-off values of nonadvanced fibrosis were set as a FIB4 score of 1.45 or a TE value of $7.4 \mathrm{kPa}^{18,19}$. FIB4 absent or mild fibrosis F0$\mathrm{F} 1<1.45$, moderate fibrosis $\mathrm{F} 2<1.45-3.25$, severe fibrosis F3 $>3.25$.

Statistical analysis: Data were analysed using the statistical package for the social sciences version 22.0 (SPSS, Chicago, IL, USA). Normal continuous variables were presented as mean \pm standard deviation. Test of significance was calculated by ordinary one-way ANOVA, the P-value was considered to be statistically significant when $<0.05$. 


\section{RESULTS}

A total of 204 NAFLD patients were distributed based on their age group, among which the majority of the patients were in the age group of $31-45$ years with $53.92 \%$ followed by $46-60$ years with $29.41 \%$ (Figure 1 ).

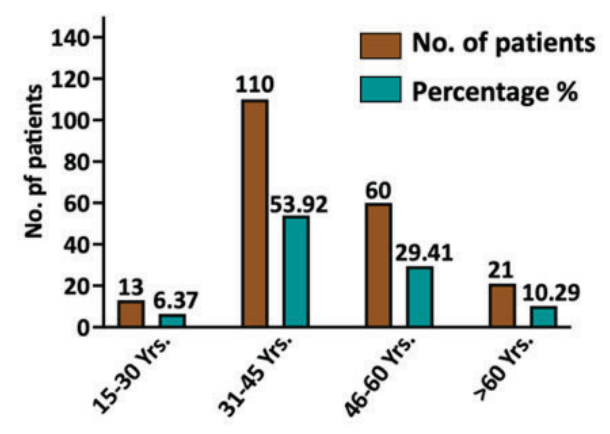

Figure 1: Distribution of NAFLD patients with age group.

Out of 304 patients, 204 (67.1\%) patients of diabetes were having CAP value $>233$ and were diagnosed as having NAFLD in which 127 (62.2\%) were female and 77 (37.7\%) were male (Figure 2).

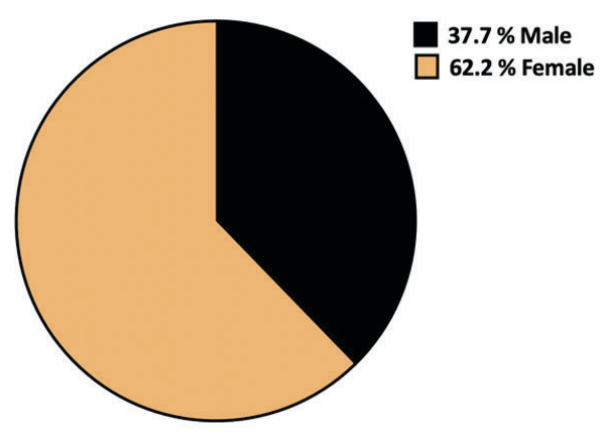

Figure 2: Gender distribution of NAFLD in diabetics.

Among 204 patients of NAFLD in study group 62 (30.39\%) were in BMI group of $<25 \mathrm{~kg} / \mathrm{m}^{2}, 90$ patients (44.11\%) were $25-30 \mathrm{~kg} / \mathrm{m}^{2}$ and $52(25.49 \%)$ were in $>30 \mathrm{~kg} / \mathrm{m} 2$ BMI group (Table 1).

Table 1: Distribution of NAFLD patients according to their BMI

\begin{tabular}{|l|l|l|}
\hline BMI & No. of patients (N=204) & Percentage of patients (\%) \\
\hline$<25$ & 62 & 30.39 \\
\hline $25-30$ & 90 & 44.11 \\
\hline$>30$ & 52 & 25.49 \\
\hline
\end{tabular}

Among 204 NAFLD patients, 65 (32\%) patients had fatty liver without fibrosis or mild fibrosis, rest 139 patients of NAFLD were divided into different categories; 92 patients were diagnosed with significant fibrosis among this, 72 patients $(78.26 \%)$ were under $<50$ years of age, 17 patients $(18.48 \%)$ were in the age group between 51-60 years of age group and only 3 patients $(3.26 \%)$ were with more than 60 years of age. 16 patients were diagnosed with severe fibrosis among this, 12 patients $(75 \%)$ were in less than 50 years of age group, 3 patients (18.75\%) were in between 51-60 years of age group and 1 patient $(6.25 \%)$ was more than 60 years. In comparison to this cirrhosis group had11 patients $(35.48 \%)$ fall under less than 50 years of age, 6 patients $(19.36 \%)$ were in 51-60 years of age group and 14 (45.16\%) patients were in age group more than 60 years which means the maximum number of patients from the study who are cirrhotic (Figure 3).

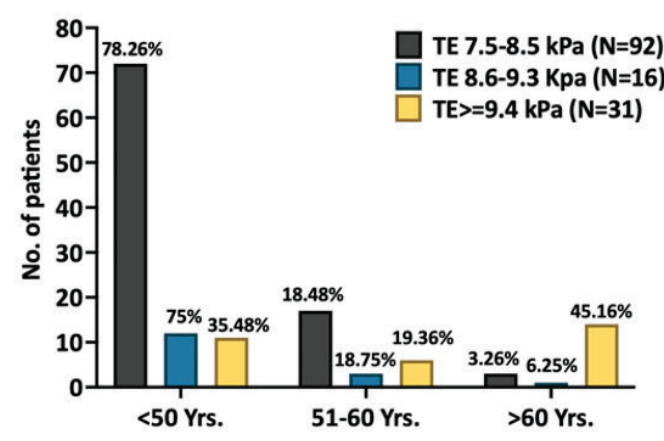

Figure 3: According to the severity of fibrosis by T.E. score age-wise distribution of NAFLD patients and their percentage.

Out of 139 patients, the mean age of fibrosis was $46.80 \pm 7.68$, the mean age of severe fibrosis was $47.94 \pm 7.02$ and the mean age of cirrhosis was $57.97 \pm 11.32$ (Figure 4).

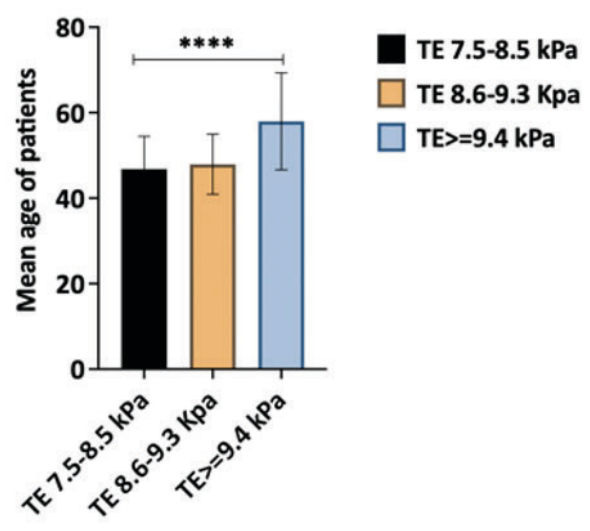

Figure 4: Mean age of patients according to the severity of fibrosis. P-value $=.0001^{*+* * *}$.

Out of 139 patients, 92(66.1\%) of the fibrosis group (TE $7.5-8.5 \mathrm{kpa}$ ) had a mean waist-hip ratio of $0.97,16$ patients $(11.5 \%)$ of severe fibrosis $(8.6-9.3 \mathrm{kpa})$ had a mean waist-hip ratio of 1.07 and rest 31 patients $(22.3 \%)$ of cirrhosis group (TE $>9.4 \mathrm{kpa}$ ) had a mean waist-hip ratio of 1.13 (Figure 5). 


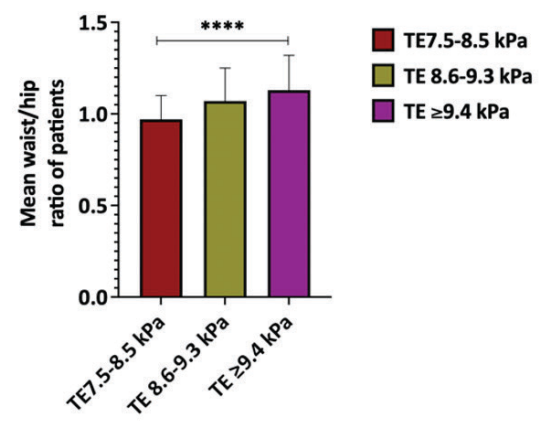

Figure 5: Mean waist/hip ratio distribution of patients according to the severity of fibrosis. P-value $=.0001^{\text {twot }}$.

Out of 139 patients, 92 patients (66.1\%) of fibrosis group (TE 7.5-8.5 kpa) showed mean BMI 29.63, 16 patients (11.5\%) of severe fibrosis (8.6-9.3 kpa) showed mean BMI 30.25 and rest 31 patients (22.3\%) of cirrhosis group (TE $>9.4 \mathrm{kpa}$ ) had mean BMI 31.94 (Figure 6).

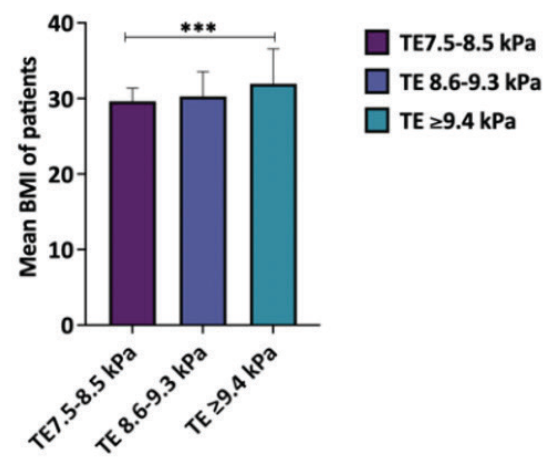

Figure 6: Mean BMI of patients according to the severity of fibrosis. P-value $=.0006^{\text {**** }}$.

Out of 139 patients, 92 patients (66.1\%) of the fibrosis group (TE 7.5-8.5 kpa) showed mean fasting sugar level of 135.88, 16 patients $(11.5 \%)$ of severe fibrosis $(8.6-9.3 \mathrm{kpa})$ showed mean fasting sugar level 157.13 and rest 31 patients (22.3\%) of cirrhosis group (TE $>9.4 \mathrm{kpa}$ ) showed mean fasting sugar 173.13 (Figure 7).

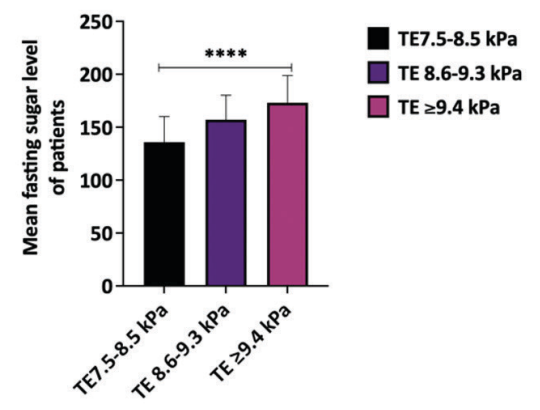

Figure 7: Mean fasting sugar level of patients according to the severity of fibrosis. P-value $=.0001^{1+* * *}$.
Out of 139 patients, 92 patients $(66.1 \%)$ of the fibrosis group (TE 7.5-8.5 kpa) had mean HbA1 cof 7.36, 16 patients $(11.5 \%)$ of severe fibrosis $(8.6-9.3 \mathrm{kpa})$ had mean $\mathrm{HbAlc}$ of 7.45 and the rest 31 patients $(22.3 \%)$ of cirrhosis group (TE $>9.4 \mathrm{kpa}$ ) had mean HbA1c of 8.05 (Figure 8).
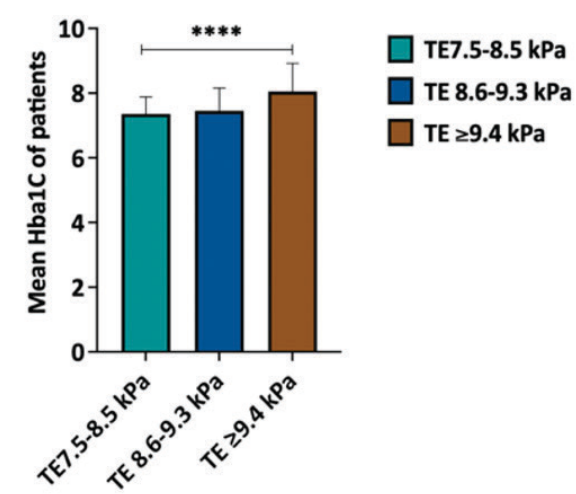

Figure 8: Mean $\mathrm{HbA} 1 \mathrm{c}$ of patients according to the severity of fibrosis $\mathrm{P}$-value $=.0001^{\text {t*t*t* }}$.

Out of 139 patients, 92 patients (66.1\%) of the fibrosis group (TE 7.5-8.5 kpa) had a mean triglyceride level of 189.49, 16 patients $(11.5 \%)$ of fibrosis $(8.6-9.3 \mathrm{kpa})$ had a mean triglyceride level of 202.63 and rest 31 patients $(22.3 \%)$ of cirrhosis group (TE $>9.4 \mathrm{kpa}$ ) had mean triglyceride level of 215.42 (Figure 9).

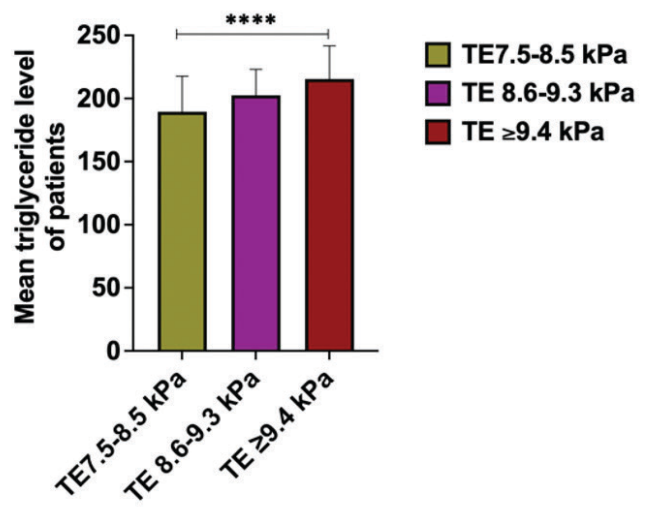

Figure 9: Mean triglyceride level of patients according to the severity of fibrosis P-value $=.0001^{\cdots * * *}$

Out of 139 patients, 92 patients (66.1\%) of the fibrosis group (TE $7.5-8.5 \mathrm{kpa}$ ) showed a mean platelet count of 136.55 , 16 patients $(11.5 \%)$ of severe fibrosis $(8.6-9.3 \mathrm{kpa})$ showed a mean platelet count of 131.38 and rest 31 patients (22.3\%) of cirrhosis group (TE $>9.4 \mathrm{kpa}$ ) had a mean platelet count of 125.52 (Figure 10). However, no significance was noticed among these groups which might be because of the small population study. 


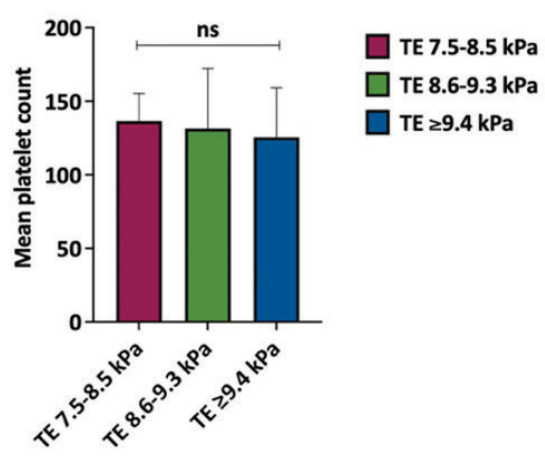

Figure 10: Mean platelet count of patients according to the severity of fibrosis $\mathrm{P}$-value $=0.117^{\mathrm{ns}}$.

Out of 139 patients, 92 patients (66.1\%) of the fibrosis group (TE $7.5-8.5 \mathrm{kpa}$ ) had a mean SGPT of 106.25, 16 patients $(11.5 \%)$ of severe fibrosis $(8.6-9.3 \mathrm{kpa})$ had a mean SGPT of 79.06 and rest 31 patients $(22.3 \%)$ of cirrhosis group (TE $>9.4 \mathrm{kpa}$ ) had mean SGPT of 90.55 (Figure 11).

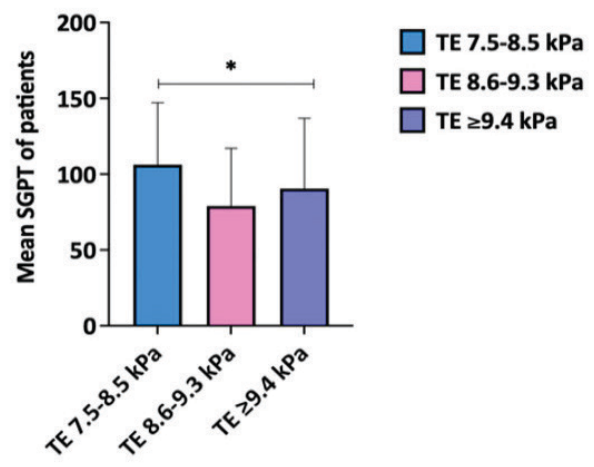

Figure 11: Mean SGPT of patients according to the severity of fibrosis. P-value $=0.02^{*}$

Out of 139 patients, 92(66.1\%) with at least significant fibrosis group (TE 7.5-8.5 kpa) were having mean SGOT 63.65 and $16(11.5 \%)$ with severe fibrosis(8.6-9.3 kpa) were having mean SGOT 50.19 and the rest 31 patients $(22.3 \%)$ of cirrhosis group (TE>9.4 kpa) having mean SGOT 52.87 .

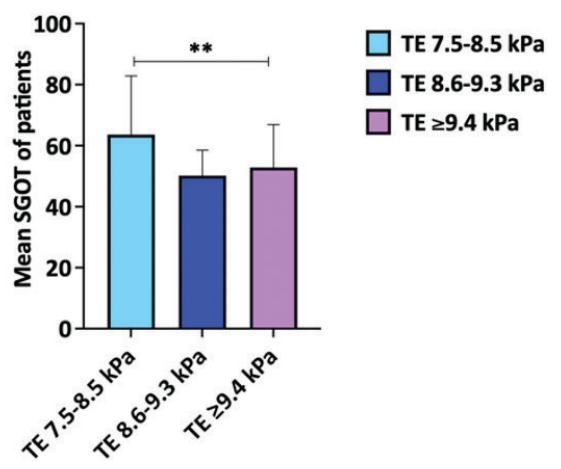

Figure 12: Mean SGOT level of patients. according to the severity of fibrosis. P-value $=.001^{\text {** }}$
Out of 304 patients, 204 patients $(67 \%)$ of diabetes were having CAP value $>233 \mathrm{~dB} / \mathrm{m}^{19}$ and were diagnosed as NAFLD, among these 204 patients, 65 patients $(32 \%)$ had fatty liver without fibrosis or mild fibrosis $(<7.4 \mathrm{kPa}), 31$ patients $(15 \%)$ had cirrhosis $(\mathrm{TE}>9.4 \mathrm{kPa})$ and rest $108(53 \%)$ patients were divided into significant fibrosis 92 patients $(45 \%)$ and severe fibrosis (TE $8.6-9.3 \mathrm{kpa}) 16$ patients $(8 \%)$ by transient elastography (Figure 13). A patient with NAFLD having significant fibrosis was also diagnosed with $\mathrm{NASH}^{20}$.

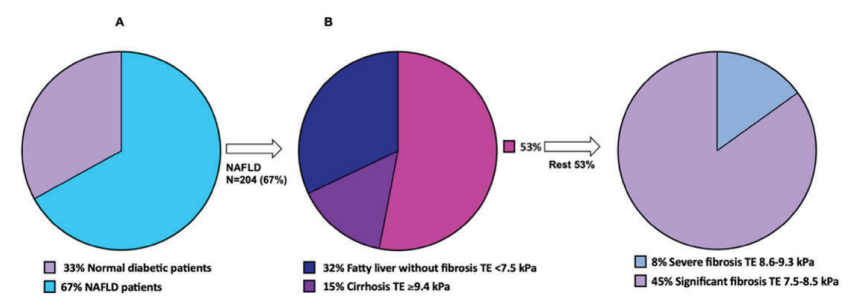

Figure 13: Hepatic involvement by TE CAP value in diabetics, $\mathrm{N}=304$ (A). Severity of hepatic fibrosis by TE scoring in NAFLD $(\mathrm{N}=204,67 \%)$ group of patients (B).

The characteristics of subjects that were identified by FIB4 and TE differed greatly (Table 2).

Table 2: Comparison of characteristics of stages of fibrosis between $\mathrm{FIB}_{4}$ and tissue elastography

\begin{tabular}{|c|c|c|c|c|c|c|c|c|c|c|c|c|}
\hline & \multicolumn{4}{|c|}{ TE 7.5-8.5 kPa (N=92) } & \multicolumn{4}{|c|}{ TE 8.6-9.3 kPa (N=16) } & \multicolumn{4}{|c|}{$T E \geq 9.4 \mathrm{kPa}(\mathrm{N}=31)$} \\
\hline & \multicolumn{4}{|c|}{ FIB score } & \multicolumn{4}{|c|}{ FIB score } & \multicolumn{4}{|c|}{ FIB score } \\
\hline & $<1.45$ & $\begin{array}{l}1.45- \\
3.25\end{array}$ & $\geq 3.25$ & P-value & $<1.45$ & $\begin{array}{l}1.45- \\
3.25\end{array}$ & $\geq 3.25$ & P-value & $<1.45$ & $\begin{array}{l}1.45- \\
3.25\end{array}$ & $\geq 3.25$ & P-value \\
\hline $\mathrm{N}=139$ & 30 & 52 & 10 & & 4 & 9 & 3 & & 5 & 14 & 12 & \\
\hline $\begin{array}{l}\text { Fatty liver } \\
(\mathrm{Y} / \mathrm{N})\end{array}$ & $14 / 16$ & $22 / 30$ & $2 / 8$ & .584 & $2 / 2$ & $4 / 5$ & $1 / 2$ & .906 & $4 / 1$ & 7/7 & $4 / 8$ & .212 \\
\hline $\begin{array}{l}\text { CAP } \\
\text { (mean } \pm S D)\end{array}$ & $\begin{array}{l}276.51 \\
\pm 23.54\end{array}$ & $\begin{array}{l}282.11 \\
\pm 19.81\end{array}$ & $\begin{array}{l}296.54 \\
\pm 40.96\end{array}$ & .078 & $\begin{array}{l}298 \\
\pm 23.7\end{array}$ & $\begin{array}{l}294.43 \\
\pm 21.45\end{array}$ & $\begin{array}{l}303.53 \\
\pm 26.67\end{array}$ & .831 & $\begin{array}{l}340.12 \\
\pm 42.24\end{array}$ & $\begin{array}{l}298.16 \\
\pm 32.88\end{array}$ & $\begin{array}{l}284.41 \\
\pm 32.87\end{array}$ & .018 \\
\hline
\end{tabular}

\section{DISCUSSION}

Out of 304 diabetic patients who underwent TE, we have found a high prevalence of NAFLD in 204 (67\%) out of which 127 (62.2\%) were females and 77 (37.7\%) were males. Majority of patients were in the age group of 31-45 years $(53.92 \%)$ followed by $46-60$ years $(29.41 \%)$. The proportion of significant fibrosis, advanced fibrosis and cirrhosis was $45 \%, 8 \%$ and $15 \%$ respectively. Our results were comparable with the previous studies conducted by Kalra S.et al., 2014 where they found 44.1 to $72 \%$ prevalence of NAFLD patients $^{21}$ and Hazlehurst et al., 2016 showed 70\% prevalence of NAFLD patients ${ }^{22}$. Based on fibroScan and CAPcut-off of $222 \mathrm{~dB} / \mathrm{m}$ Kwok showed 72.8\%occurrence of NAFLD in diabetic patients and another group of Lee-lee lai from Malaysia based on the same parameter but a different CAP cut-off of $263 \mathrm{~dB} / \mathrm{m}$ showed $72.4 \%$ occurrence of NAFLD patients $^{23,24}$. The CAP cut-off we used in this study was $>233$ $\mathrm{dB} / \mathrm{m}$ which was seen to be compatible with previously 
reported literature. Wong V et al.,2018 recommended that by the fibroscan, CAP can be measured simultaneously with liver stiffness and thus may be used to diagnose NAFLD by confirming the presence of steatosis and assessing its severity. ${ }^{25}$

In addition, it is important to note that among diabetic patients with $\mathrm{BMI}<25 \mathrm{~kg} / \mathrm{m} 2,30.39 \%$ of patients still had NAFLD. In previous studies about NAFLD, non-obese patients usually have other components of metabolic syndrome ${ }^{26}$. The presence of diabetes itself is one of the components for metabolic syndrome and the prevalence of metabolic syndrome was high in our study population of diabetics with NAFLD. With the increasing prevalence of obesity, diabetes mellitus, and metabolic syndrome, the general population of NAFLD has become the most common cause of chronic liver disease worldwide. ${ }^{27}$

Out of 139 patients with significant fibrosis and cirrhosis, the mean age for significant fibrosis was (46.80 \pm 7.68$)$, the mean age for severe fibrosis was (47.94 \pm 7.02$)$ and the mean age for cirrhosis was $(57.97 \pm 11.32)$. TE value is more in higher age group patients which signifies higher chances of cirrhosis in old age patients and thus it can be concluded that the severity of NAFLD increases with age. Several groups have published systematic reviews on risk factors like age as an independent factor in the development of advanced fibrosis in patients with NASH. ${ }^{28-31}$

This study shows as the waist-hip ratio increases the severity of NAFLD defined by TE from significant fibrosis towards cirrhosis also increases, this result was comparable with the study done by Cheng PN et al., 2016 where they have linked abnormal waist circumference to a higher prevalence of NAFLD. ${ }^{32}$

Our data reports that as mean BMI increases the severity of the disease also increases which is statistically significant with a $\mathrm{p}$-value $=0.006$. This finding of ours was similar to the previous study done by Yen YH et al., 2018 where they have stated BMI as an independent factor correlated with clinically relevant fibrosis in patients with metabolic risk factors linked with NAFLD. ${ }^{33}$

Increased fasting blood sugar, high $\mathrm{HbA1C}$, Increase triglyceride value increases the severity of the disease from significant fibrosis towards cirrhosis. Increased fasting blood sugar levels and increased serum triglyceride for a longer duration appeared as important risk factors for the development of a NAFLD. Mohan V et al., 2009 showed an increasing prevalence of NAFLD with the increase in severity of glucose intolerance. ${ }^{34}$ Another group of Alagesan et al., 2019 also revealed that poor glycemic control has a significant correlation with the increase in liver stiffness ${ }^{35}$ which was also supported by several other studies. ${ }^{36-39}$ Lavekar Anurag et al., 2015 confirms that the increased BMI, metabolic syndrome, increased fasting blood glucose and serum triglycerides are potentially strong indicators of NAFLD. ${ }^{40}$

As the severity of hepatic changes increases toward more severe disease, the platelet count decreases hence low platelet count is a risk factor for the severity of NAFLD. Lower platelet levels expect a more extreme hepatic fibrosis. Witters et al., 2008 mentioned thrombocytopenia as a marked feature of chronic liver disease and cirrhosis. ${ }^{41}$ Jaafar et al., 2019 stated that the majority of liver function tests did not reflect severe liver status in diabetic patients, except $\gamma$-glutamyl transferase and prothrombin time-international normalized ratio $(0.08$ and 0.00$){ }^{42}$ Similar observations were made by Wong V et al.,2006 where they have reported prioritizing the metabolic status of the patient over the serum transaminases when determining the severity of the liver fibrosis. ${ }^{43}$ One more study showed a high proportion of patients with severe liver fibrosis and cirrhosis had normal ALT and AST. ${ }^{44}$ Our study shows that with increased fibrosis score or liver cirrhosis in patients ALT and AST don't need to also increase. As the fibrosis increases AST and ALT may even be normal which was comparable with the previous findings.

Out of 304 patients, NAFLD was present in $204(67 \%)$ patients defined by transient elastography. But out of 304 patients, only $60(43.1 \%)$ patients were having fatty liver disease detected by ultrasonography, which is comparable with a previous study done by Amarapurkar D et al., 2007.45 This is why transient elastography can detect earlier hepatic changes as fatty liver as compared to ultrasonography.

FIB4 and TE yielded a different type of fibrosis distribution in the same diabetic population. The characteristics of subjects that were identified by FIB4 and TE differed greatly. This result indicates that these two methods are not interchangeable. In this study among three TE defined fibrosis stages, CAP value is higher as fibrosis stages become severe especially in those subjects with FIB $4<1.45$ in TE defined advanced fibrosis. The results in this study indicated the possible role of steatosis in the measurement of liver stiffness by TE, especially combined with low FIB4 score subjects. The presence of fatty liver is strongly associated with TE defined advanced fibrosis rather than FIB4 defined advanced fibrosis. In those subjects TE defined advanced fibrosis, higher CAP values and increased incidence of fatty liver was observed in subjects with FIB- $4<1.45$, indicates that TE may have the advantage over the FIB4 to detect the involvement of non-alcoholic fatty liver disease in fibrosis progression of patients with diabetes thus TE is probably better than FIB4 for evaluating fatty liver leading to liver fibrosis for early detection of NAFLD in patients of diabetes. This result was in correlation with a previous study done by Pin-Nan cheng et al., 2018 where they have stated that FIB4 scoring was affected by age but not TE. ${ }^{46}$ TE had also an advantage in the identification of NAFLD in advanced stages of fibrosis. 
Summary: Severity of NAFLD was associated with an increase in mean age, mean waist-hip ratio, mean BMI, mean fasting blood sugar, mean $\mathrm{HbA} 1 \mathrm{C}$ and mean triglyceride. In comparison to this lower mean platelet value expect more severe fibrosis, and there is no direct correlation with SGOT and SGPT with the severity of disease and greater variability in a different group of stages of fibrosis. The higher stage of fibrosis was observed in older age group individuals and this was comparable to the study done by Coppell KJ et al., 2015 and confirmed that this result connects age to an increased risk of severe hepatic fibrosis, HCC and Diabetes mellitus. ${ }^{47}$ It was observed that impaired glycaemic control leads to more severe disease and explained by the fact that the liver is an insulin-sensitive tissue that is susceptible to the effects of hyperglycaemia induced oxidative stress leading to liver tissue injury. Lipotoxicity can appear as a result of the accumulation of fats in non-adipose tissues and can develop secondary to insulin resistance in patients with NAFLD. It plays a pivotal role in the progression of a milder form of NAFLD to NASH which was also revealed in our study that patients with cirrhosis have a higher level of mean triglyceride in comparison to significant fibrosis patients. Advanced fibrosis can precede portal hypertension inducing thrombocytopenia due to increased sequestration and destruction of platelets in the enlarged spleen.$^{48}$ Lower platelet levels predict a more extreme hepatic fibrosis. Patients with liver cirrhosis have independent mean ALT and AST levels.

\section{CONCLUSION}

The prevalence of NAFLD is very high in diabetic patients. The main factor in the management of NAFLD is the earliest detection of the disease so that we can modify the risk factor in the earliest stage and prevent further damage of the liver by strict follow-up, lifestyle changes, blood sugar control, weight reduction and prevent complication. Transient elastography (TE) is a better non-invasive tool for the earliest detection of NAFLD without any risk for fatal complications of invasive procedure and to detect liver fibrosis earlier than FIB4.

Limitation of this study: This study has its limitations like we don't have an XL probe for fibroScan (TE) examination on patients with BMI more than $28 \mathrm{~kg} / \mathrm{m}^{2}$ and lack of liver biopsy for confirmation of our fibroScan results. Our results may not be generalised to all primary care level patients and in patients with diabetes at the hospital. A study with a larger sample size may lead to more accurate results.

Abbreviations: Fibrosis 4 (FIB4), Transient elastography (TE), Non-alcoholic fatty liver disease (NAFLD), Glycated haemoglobin(HbAlc), Body mass index (BMI), Nonalcoholic steatohepatitis (NASH), Controlled attenuation parameter (CAP), American association for the study of liver diseases (AASLD), European association for the study of the liver (EASL), Complete blood count (CBC), Serum alanine aminotransferase(ALT), Aspartate aminotransferase (AST), Gamma-glutamyl transpeptidase (GGT), Alkaline phosphatase (AlP), Diabetes mellitus (DM), Liver stiffness measurement (LSM), hepatitis B surface antigen (HBsAg), Hepatitis $\mathrm{C}$ virus (HCV), Breakpoints Pressure ( $\mathrm{kPa}$ ), Serum glutamic pyruvic transaminase (SGPT), Serum glutamic-oxaloacetic transaminase (SGOT) and Ultrasonography (USG).

\section{ACKNOWLEDGEMENT}

I am grateful to my guide Dr. (Prof.) Balvirsingh, head of P.G. department of medicine, Sarojini Naidu medical college Agra for letting me conduct this study and helping me in every way possible.

Authors contribution: Data curation, methodology, visualization, writing original draft, reviewing and editing: Dr. Vipin Singh.Supervision, reviewing and editing: Dr. (Prof) Balvirsingh.

Conflict of interest: Authors have declared that no conflict of interest exists.

Funding: This study was carried out in SN medical college Agra with no external funding source.

\section{REFERENCES}

1. Danaei G, Finucane M, Lu Y, Singh G, Cowan M, Paciorek C et al. National, regional, and global trends in fasting plasma glucose and diabetes prevalence since 1980: systematic analysis of health examination surveys and epidemiological studies with 370 country-years and 2.7 million participants. The Lancet. 2011jul 2;378(9785):31-40.

2. Torres, D.M. Non-Alcoholic Fatty Liver Disease. In: Feldman, M, Friedman, L.S, Brandt, L.J (eds.) Sleisenger and Fordtran's Gastrointestinal and liver disease pathophysiology/diagnosis/ management. Philadelphia: Elsevier Saunders; 2006. p. 177299.

3. Trombetta M, SpiazziG, Zoppini G, Muggeo M. Review article: type 2 diabetes and chronic liver disease in the Verona diabetes study. Aliment Pharmacol and Ther. 2005 Nov;22(s2):24-27.

4. Hultcrantz R, Glaumann H, Lindberg G, H:son Nilsson L. Liver Investigation in 149 Asymptomatic Patients with Moderately Elevated Activities of Serum: Aminotransferases. Scand J Gastroenteol. 1986 Jan 1;21(1):109-113.

5. Harris M, Flegal K, Cowie C, Eberhardt M, Goldstein D, Little $\mathrm{R}$ et al. Prevalence of Diabetes, Impaired Fasting Glucose, and Impaired Glucose Tolerance in U.S. Adults: The Third National Health and Nutrition Examination Survey, 1988-1994. Diabetes Care. 1998 Apr 1;21(4):518-524.

6. Caldwell S, Oelsner D, Iezzoni J, Hespenheide E, Battle E, Driscoll C. Cryptogenic cirrhosis: clinical characterization and risk factors for underlying disease. Hepatology. 1999 Mar;29(3):664-669.

7. Ludwig J, Viggiano TR, Mcgill DB, Oh BJ. Nonalcoholic steatohepatitis: Mayo Clinic experiences with a hitherto unnamed disease. Mayo ClinProc.1980 Jul;55(7): 434-438. 
8. Powell E, Cooksley W, Hanson R, Searle J, Halliday J, Powell W. The natural history of nonalcoholic steatohepatitis: A followup study of forty-two patients for up to 21 years. Hepatology. 1990 Jan;11(1):74-80.

9. Lee R. Nonalcoholic steatohepatitis: A study of 49 patients. Human Pathology. 1989 Jun;20(6):594-598.

10. Itoh S, Yougel T, Kanagoe K. Comparison between nonalcoholic steatohepatitis and alcoholic hepatitis. Am J Gastroenterol.1987 Jul;82(7):650-4.

11. Diehl A, Goodman Z, Ishak K. Alcohollike liver disease in nonalcoholics. Gastroenterology. 1988; 95(4):1056-1062.

12. Pinto H, Baptista A, Camilo M, Valente A, Saragoça A, de Moura M. Nonalcoholic steatohepatitis. DigestDis Sci. 1996Jan;41(1):172-179.

13. Silverman JF, O'Brien KF, Long S, Leggett N, Khazanie PG, Pories WJ, Norris HT, Caro JF. Liver pathology in morbidly obese patients with and without diabetes. AmJ Gastroenterol.1990 Oct ;85(10):1349-55

14. Chalasani N, Younossi Z, Lavine J, Charlton M, Cusi K, Rinella $\mathrm{M}$ et al. The diagnosis and management of nonalcoholic fatty liver disease: Practice guidance from the American Association for the Study of Liver Diseases. Hepatology. 2017 Jan;67(1):328357.

15. EASL-EASD-EASO Clinical Practice Guidelines for the Management of Non-Alcoholic Fatty Liver Disease. Obesity Facts. 2016;9(2):65-90.

16. Tuong T, Tran D, Phu P, Hong T, Chu Dinh T, Chu D. NonAlcoholic Fatty Liver Disease in Patients with Type 2 Diabetes: Evaluation of Hepatic Fibrosis and Steatosis Using Fibroscan. Diagnostics. 2020Mar 14;10(3):159.

17. Foucher J. Diagnosis of cirrhosis by transient elastography (FibroScan): a prospective study. Gut. 2006 Mar;55(3):403-408.

18. Vallet-Pichard A, Mallet V, Nalpas B, Verkarre V, Nalpas A, Dhalluin-Venier V, et al. FIB-4: an inexpensive and accurate marker of fibrosis in HCV infection. comparison with liver biopsy and fibrotest. Hepatology. 2007 Jul;46(1):32-6.

19. Castéra L, Vergniol J, Foucher J, Le Bail B, Chanteloup E, Haaser M, et al. Prospective comparison of transient elastography, Fibrotest, APRI, and liver biopsy for the assessment of fibrosis in chronic hepatitis C. Gastroent. 2005 Feb;128(2):343-50.

20. Adams LA, Feldstein AE. Non-invasive diagnosis of nonalcoholic fatty liver and nonalcoholic steatohepatitis. J Dig Dis. 2011 Feb;12(1):10-6.

21. Kalra S, Vithalani M, Gulati G, Kulkarni CM, Kadam Y, Pallivathukkal J, et al. Study of the prevalence of nonalcoholic fatty liver disease (NAFLD) in type 2 diabetes patients in India (SPRINT). J Assoc Physicians India. 2013 Jul;61(7):448-53.

22. Hazlehurst JM, Woods C, Marjot T, Cobbold JF, Tomlinson JW. Non-alcoholic fatty liver disease and diabetes. Metabolism. 2016 Aug 1;65(8):1096-108.

23. Kwok R, Choi KC, Wong GL, Zhang Y, Chan HL, Luk AO, et al. Screening diabetic patients for non-alcoholic fatty liver disease with controlled attenuation parameter and liver stiffness measurements: a prospective cohort study. Gut. 2016 08;65(8):135968.

24. Lai LL, Wan Yusoff WNI, Vethakkan SR, Nik Mustapha NR, Mahadeva S, Chan WK. Screening for non-alcoholic fatty liver disease in patients with type 2 diabetes mellitus using transient elastography. J Gastroenterol Hepatol. 2019 Aug;34(8):1396403.

25. Wong VW, Chan WK, Chitturi S, Chawla Y, Dan YY, Duseja A, et al. Asia-Pacific Working Party on Non-alcoholic Fatty Liver Disease guidelines 2017-Part 1: Definition, risk factors and assessment. J Gastroenterol Hepatol. 2018 Jan;33(1):70-85.
26. Das K, Das K, Mukherjee PS, Ghosh A, Ghosh S, Mridha AR, et al. Nonobese population in a developing country has a high prevalence of nonalcoholic fatty liver and significant liver disease. Hepatology. 2010 May;51(5):1593-602.

27. Angulo P, Hui JM, Marchesini G, Bugianesi E, George J, Farrell GC, et al. The NAFLD fibrosis score: a noninvasive system that identifies liver fibrosis in patients with NAFLD. Hepatology. 2007 Apr;45(4):846-54.

28. Fan JG, Zhu J, Li XJ, Chen L, Li L, Dai F, et al. Prevalence of and risk factors for fatty liver in a general population of Shanghai, China. J Hepatol. 2005 Sep;43(3):508-14.

29. Hu X, Huang Y, Bao Z, Wang Y, Shi D, Liu F, et al. Prevalence and factors associated with nonalcoholic fatty liver disease in Shanghai work-units. BMC Gastroenterol. 2012 Sep 14; 12:123.

30. Eguchi Y, Hyogo H, Ono M, Mizuta T, Ono N, Fujimoto K, et al. Prevalence and associated metabolic factors of nonalcoholic fatty liver disease in the general population from 2009 to 2010 in Japan: a multicenter large retrospective study. J Gastroenterol. 2012 May;47(5):586-95.

31. Argo CK, Northup PG, Al-Osaimi AM, Caldwell SH. Systematic review of risk factors for fibrosis progression in non-alcoholic steatohepatitis. J Hepatol. 2009 Aug;51(2):371-9.

32. Cheng PN, Chiu YC, Chiu HC, Chien SC. The Application of Liver Stiffness Measurement in Residents Without Overt Liver Diseases Through a Community-Based Screening Program. Medicine (Baltimore). 2016 Mar;95(12): e3193.

33. Yen YH, Chang KC, Tsai MC, Tseng PL, Lin MT, Wu CK, et al. Elevated body mass index is a risk factor associated with possible liver cirrhosis across different etiologies of chronic liver disease. J Formos Med Assoc. 2018 Apr;117(4):268-75.

34. Mohan V, Farooq S, Deepa M, Ravikumar R, Pitchumoni CS. Prevalence of non-alcoholic fatty liver disease in urban south Indians in relation to different grades of glucose intolerance and metabolic syndrome. Diabetes Res Clin Pract. 2009 Apr;84(1):84-91.

35. Alagesan SK. Assessment of liver involvement in type 2 diabetes mellitus using fibroscan ${ }^{\circledR}$ and correlation with risk factors. Int J Adv Med.2019 May;6(3):594.

36. Gupte P, Amarapurkar D, Agal S, Baijal R, Kulshrestha P, Pramanik $\mathrm{S}$, et al. Non-alcoholic steatohepatitis in type 2 diabetes mellitus. J Gastroenterol Hepatol. 2004 Aug;19(8):854-8.

37. Banerjee S, Ghosh US, Dutta S. Clinicopathological profile of hepatic involvement in type-2 diabetes mellitus and its significance. J Assoc Physicians India. 2008 Aug; 56:593-9.

38. Duseja A, Das A, Das R, Dhiman RK, Chawla Y, Bhansali A, et al. The clinicopathological profile of Indian patients with nonalcoholic fatty liver disease (NAFLD) is different from that in the West. Dig Dis Sci. 2007 Sep;52(9):2368-74.

39. Duseja A, Das A, Dhiman RK, Chawla YK, Das R, Bhadada S, et al. Indian patients with nonalcoholic fatty liver disease presenting with raised transaminases are different at presentation. World J Gastroenterol. 2007 Jan 28;13(4):649-50.

40. Anurag L, Aniket S, Shalik J, Amarja L, Dhananjay R, Sachin J. Non-alcoholic fatty liver disease prevalence and associated risk factors--A study from rural sector of Maharashtra. Trop Gastroenterol. 2015 Jan-Mar;36(1):25-30.

41. Witters P, Freson K, Verslype C, Peerlinck K, Hoylaerts M, Nevens F, et al. Review article: blood platelet number and function in chronic liver disease and cirrhosis. Aliment PharmacolTher. 2008 Jun 1;27(11):1017-29.

42. Jaafar RF, Hajj Ali AM, Zaghal AM, Kanso M, Habib SG, Halaoui AF, et al. Fibroscan and low-density lipoprotein as determinants of severe liver fibrosis in diabetic patients with nonalcoholic fatty liver disease. Eur J Gastroenterol Hepatol. 2019 Dec;31(12):1540-4. 
43. Wong VW, Hui AY, Tsang SW, Chan JL, Wong GL, Chan AW, et al. Prevalence of undiagnosed diabetes and postchallengehyperglycaemia in Chinese patients with non-alcoholic fatty liver disease. Aliment PharmacolTher. 2006 Oct 15;24(8):1215-22.

44. Pathik P, Ravindra S, Ajay C, Prasad B, Jatin P, Prabha S. Fibroscan versus simple noninvasive screening tools in predicting fibrosis in high-risk nonalcoholic fatty liver disease patients from Western India. Ann Gastroenterol. 2015 Apr-Jun;28(2):281-6.

45. Amarapurkar D, Kamani P, Patel N, Gupte P, Kumar P, Agal S, et al. Prevalence of non-alcoholic fatty liver disease: populationbased study. Ann Hepatol. 2007 Jul-Sep;6(3):161-3.
46. Cheng PN, Chiu HC, Chiu YC, Chen SC, Chen Y. Comparison of FIB-4 and transient elastography in evaluating liver fibrosis of chronic hepatitis C subjects in community. PLoS One. 2018;13(11): e0206947.

47. Coppell KJ, Miller JC, Gray AR, Schultz M, Mann JI, Parnell WR. Obesity and the extent of liver damage among adult New Zealanders: findings from a national survey. Obes Sci Pract. 2015 12;1(2):67-77.

48. Wai CT, Greenson JK, Fontana RJ, Kalbfleisch JD, Marrero JA, Conjeevaram HS, et al. A simple noninvasive index can predict both significant fibrosis and cirrhosis in patients with chronic hepatitis C. Hepatology. 2003 Aug;38(2):518-26. 